University of Warwick institutional repository: http://go.warwick.ac.uk/wrap This paper is made available online in accordance with publisher policies. Please scroll down to view the document itself. Please refer to the repository record for this item and our policy information available from the repository home page for further information.

To see the final version of this paper please visit the publisher's website. Access to the published version may require a subscription.

Author(s): $\quad$ A. A. Davis, R. G. Jones, G. Falkenberg, L. Seehofer, R. L. Johnson, and C. F. McConville

Article Title: Evidence from scanning tunneling microscopy in support of a structural model for the $\operatorname{lnSb}(001)-c(8 \times 2)$ surface Year of publication: 1999

Link to published version: http://dx.doi.org/10.1063/1.124877

Publisher statement: None 


\title{
Evidence from scanning tunneling microscopy in support of a structural model for the $\operatorname{InSb}(001)-c(8 \times 2)$ surface
}

\author{
A. A. Davis and R. G. Jones \\ School of Chemistry, University of Nottingham, Nottingham NG7 2RD, United Kingdom \\ G. Falkenberg, L. Seehofer, and R. L. Johnson \\ II-Institut fur Experimental Physik, Universitat Hamburg, D-22761 Hamburg, Germany \\ C. F. McConville \\ Department of Physics, University of Warwick, Coventry CV4 7AL, United Kingdom
}

(Received 13 May 1999; accepted for publication 23 July 1999)

\begin{abstract}
In this letter we present evidence from scanning tunneling microscopy studies in support of a recently proposed structural model for the indium-terminated $c(8 \times 2)$ surface of $\operatorname{InSb}(001)$. This structural model, by Norris and co-workers, is based on a surface x-ray diffraction study [Surf. Sci. 409, 27 (1998)], and represents a significant departure from previously suggested models for the $c(8 \times 2)$ reconstruction on any $(001)$ surface of the common III-V semiconductor materials. Although filled state images of the $\operatorname{InSb}(001)-c(8 \times 2)$ surface have previously been published, empty states image of sufficient quality to extract any meaningful information have not previously been reported. The observations are in excellent agreement with the recently proposed model for this surface reconstruction. (C) 1999 American Institute of Physics. [S0003-6951(99)03138-1]
\end{abstract}

In contrast to most III-V semiconductor surface reconstructions that are group $\mathrm{V}$ terminated, and whose structures have been determined, there has been considerable debate over the exact composition and atomic structure of the outermost layers of the group III terminated reconstructions. ${ }^{1,2}$ This has clearly been demonstrated by the controversy surrounding the $\mathrm{InSb}(001)-c(8 \times 2)$ surface reconstruction. ${ }^{3-5}$

The initial model for the $\operatorname{InSb}(001)-c(8 \times 2)$ surface was proposed by John et al., ${ }^{6}$ based on high energy electron diffraction, soft x-ray photoemission data and comparison with the same surface reconstruction previously observed by Biegleson et al. for $\mathrm{GaAs}(001)$ using scanning tunneling microscopy (STM). ${ }^{7}$ This model had the surface terminated with 0.75 monolayer (ML) of indium [where $1 \mathrm{ML}$ is the In concentration in a $(1 \times 1)$ unreconstructed In-terminated surface], made up of blocks of three In dimers and a missing dimer forming a $(4 \times 2)$ block with the dimer bonds parallel to the [110] direction, on top of a complete Sb layer. The $c(8 \times 2)$ unit mesh was then formed by staggering the rows of $(4 \times 2)$ blocks in the [110] direction by $\times 1$.

A previous STM study of the $c(8 \times 2)$ surface was performed by Schweitzer et al. ${ }^{3}$ on an $\mathrm{InSb}(001)$ sample prepared in situ by cycles of low energy ion bombardment and annealing. Filled state images indicated double rows of bright dots aligned along the [110] direction, separated by darker regions in which no structure could be resolved. No empty state images were recorded. These authors could only identify a $(4 \times 1)$ unit mesh in their STM images, which conflicted with the $c(8 \times 2)$ translational symmetry observed in the low energy electron diffraction (LEED) pattern. This apparent conflict was resolved by attributing the pairs of bright rows in the STM image to tunneling from filled lone-

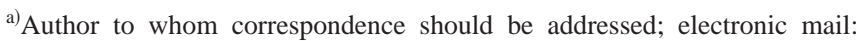
C.F.McConville@warwick.ac.uk
}

pair orbitals of the $\mathrm{Sb}$ atoms in the second layer at the location of the missing In dimers in the top layer of the structure. Although agreeing with the available data at the time, this assignment was later shown to be incorrect.

Varekamp et al. ${ }^{5}$ also studied the $\operatorname{InSb}(001)-c(8 \times 2)$ surface by STM, again prepared by low energy ion bombardment $(500 \mathrm{eV})$ followed by annealing to $350-400{ }^{\circ} \mathrm{C}$. These authors were able to determine a true $c(8 \times 2)$ symmetry in their filled states images. They also observed that the spot separation in the double rows was not exactly one surface lattice constant in the [110] direction, but oscillated about this value. This alternating compression and expansion resulted in an observed two-fold periodicity, creating a (4 $\times 2)$ unit mesh instead of the previously observed $(4 \times 1)$ unit mesh. ${ }^{3}$ They also observed a $(4 \times 2)$ unit mesh in a relatively low quality empty states image, but there was insufficient data from which to derive any detailed structural information. ${ }^{5}$ The observation of the true $c(8 \times 2)$ unit mesh does, however, indicate that the double rows of bright spots cannot be due to second layer $\mathrm{Sb}$ atoms. Although noting this fact, Varekamp et al. were unable to suggest an alternative structural model for the surface that agreed with their STM images.

The most recent structural model for this $\operatorname{InSb}(001)-c(8 \times 2)$ surface, proposed by Norris and coworkers, is based on a surface x-ray diffraction study. ${ }^{8}$ This model is characterized by chains of metallic bonded indium atoms extending along the [110] direction, separated by pairs of $\mathrm{Sb}$ dimers, with both the metallic indium and the $\mathrm{Sb}$ dimers residing on top of an $\mathrm{Sb}$ terminated bulk structure. In this letter we present evidence from high resolution filled and empty state STM images which strongly support this structural model for the $\operatorname{InSb}(001)-c(8 \times 2)$ surface.

The experimental work was carried out at Hamburg University, Germany, using a large ultrahigh vacuum system 
equipped with comprehensive sample preparation and analysis facilities (including LEED). Images were recorded with a commercial STM I (Omicron, GmbH). The $n$-type doped $\left(n \sim 2 \times 10^{16} \mathrm{~cm}^{-3}\right.$ ) $\mathrm{InSb}(001)$ samples (Wafer Technology, UK) were chemically etched prior to insertion in vacuum and cleaned in situ by cycles of low energy $\mathrm{Ar}^{+}$ion bombardment $\left(500 \mathrm{eV}\right.$, with the ion beam at $45^{\circ}$ to the surface normal) followed by annealing to $350{ }^{\circ} \mathrm{C}$ for up to $30 \mathrm{~min}$. The resulting surface, which displayed a clear $c(8 \times 2)$ LEED pattern, was checked for lack of contaminants using Auger electron spectroscopy prior to transfer to the STM, where all the imaging occurred at room temperature. Typical imaging conditions for both filled and empty states used bias voltages between $\pm 2 \mathrm{~V}$ with tunneling currents of 2-3 nA.

The STM image shown in Fig. 1 is a filled states image $(70 \AA \times 70 \AA)$ of the $\operatorname{InSb}(001)-c(8 \times 2)$ surface following three cycles of $\mathrm{Ar}^{+}$bombardment and annealing. Rows of bright features (marked A) are observed along the [110] direction [with a separation of $4.5 \pm 0.2 \AA(\times 1)$ between features and 19.6 $\pm 0.2 \AA(\times 4)$ between adjacent rows]. Between these bright rows are two more rows of features (marked B) that are somewhat less intense and evenly distributed along the [110] direction. This image, recorded with a bias voltage of $-2.0 \mathrm{~V}$ and a tunnel current of $1.67 \mathrm{nA}$, differs from the previously published work of Schweitzer et al.,$^{3}$ but is similar to the image produced by Varekamp et al. ${ }^{5}$ Marked on Fig. 1 are a series of $(4 \times 2)$ blocks which have been arranged to form the overall $c(8 \times 2)$ unit mesh seen in LEED. Within each $(4 \times 2)$ block pairs of $B$ features have been joined with a short line.

Figure 2 shows an empty states image $(90 \AA \times 90 \AA)$ of the same $\operatorname{InSb}(001)-c(8 \times 2)$ surface. The image was recorded with a sample bias of $+1.1 \mathrm{~V}$ and a tunnel current of $3.2 \mathrm{nA}$, although similar images were also recorded at slightly lower tunnel currents. As in the filled states image, a series of bright features are seen (again marked A) aligned along the [110] direction with the same $4.5 \pm 0.2 \AA(\times 1)$ separation. However, the structure observed between adjacent rows of bright dots is now very different. Bright, elongated features, marked $\mathrm{C}$ and aligned parallel to the [1 $\overline{\mathbf{1}} 0]$ direction are seen linking adjacent rows of $\mathrm{A}$ features (rather like rungs on a ladder), with a separation of $9.0 \pm 0.2 \AA$ $(\times 2)$, and with each set of rungs shifted by $\times 1(4.5 \AA)$ with respect to the adjacent rows. In addition between each rung, less well resolved features appear (marked D) the overall appearance being of a faint row of diffuse features halfway between and halfway along each rung. This image clearly shows the full $c(8 \times 2)$ unit mesh expected from the LEED pattern, but not observed directly in previous STM studies, as empty states image of sufficient quality to extract any meaningful information have not previously been reported for this surface reconstruction.

When both the filled and empty state images are considered in terms of the structural model proposed by Norris and co-workers $^{8}$ (shown in Fig. 3), it is possible to assign the observed features in both images. The dominant features in both the filled and empty state images are the rows of bright features marked A, aligned along the [110] direction. Because of their prominence at both positive and negative bias voltages, these features are attributed to states associated Downloaded 06 Jul 2009 to 137.205 .202 .8 . Redistribution subject

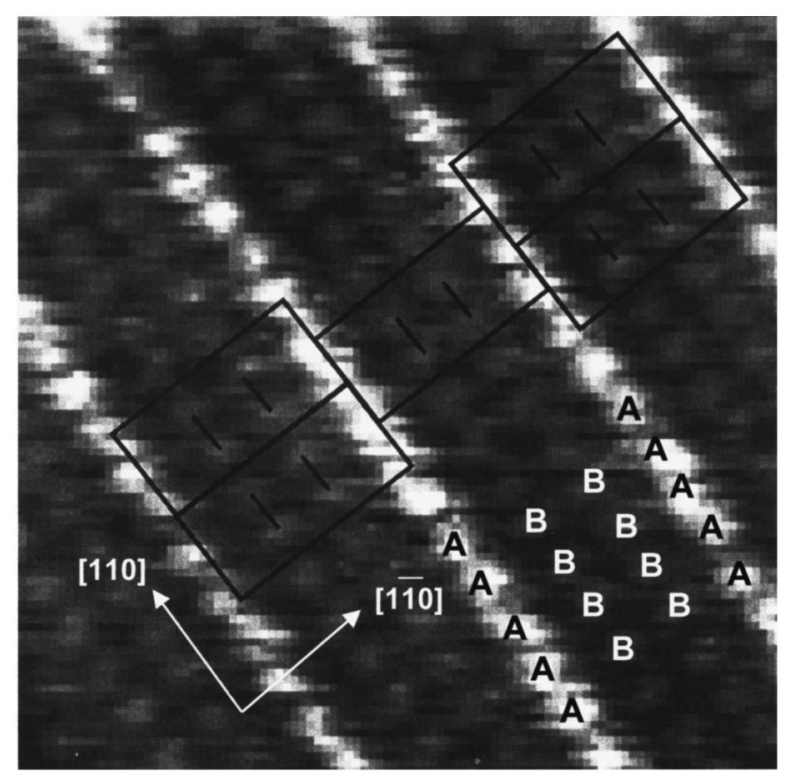

FIG. 1. Filled states STM image ( $70 \AA \times 70 \AA)$ of the $\operatorname{InSb}(001)-c(8 \times 2)$ surface showing the $(4 \times 2)$ unit mesh arranged to form the overall $c(8$ $\times 2)$ translational symmetry. Each unit mesh spans the adjacent rows of indiums (marked as A) aligned along the [110] direction and contains two $\mathrm{Sb}-\mathrm{Sb}$ dimer pairs (marked by the dash, $\mathrm{B}-\mathrm{B}$ ) with the dimer direction also along the [110] direction. The image was recorded with a sample bias of $-2.0 \mathrm{~V}$ and a tunnel current of $1.67 \mathrm{nA}$.

with metallic bonding in the In rows. ${ }^{8}$ Metallic bonding generally requires high coordination numbers and the arrangement of what is effectively a triple row of In atoms along the [110] direction, separated by the same interatomic distance observed in indium metal, satisfies this condition. We therefore identify the A features in Figs. 1 and 2 as due to the rows of indium atoms shown in Fig. 3.

If one now considers the filled states image in Fig. 1, the structure appears to be $(4 \times 1)$. However, closer scrutiny of

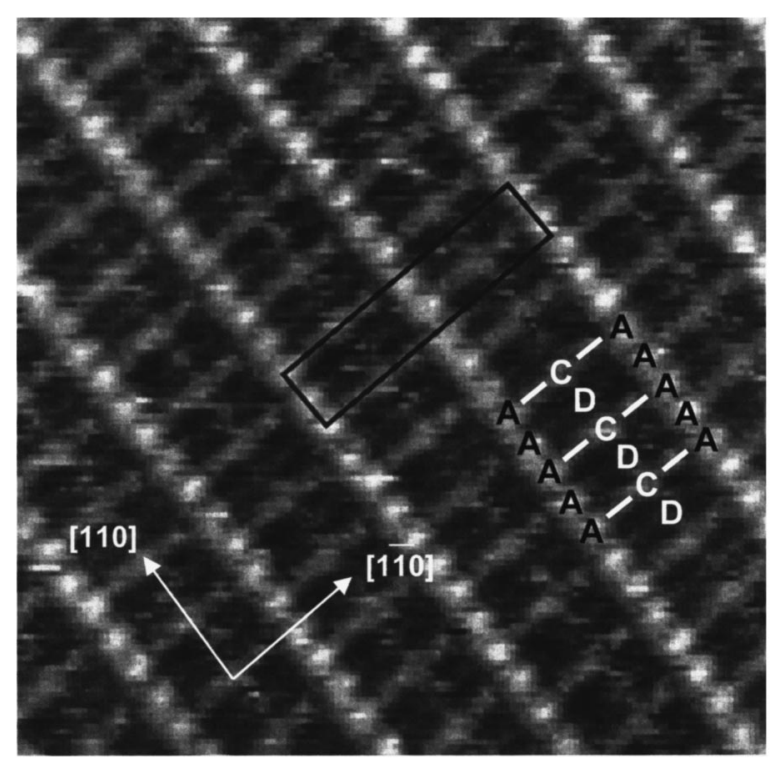

FIG. 2. Empty states STM image $(90 \AA \times 90 \AA)$ of the $\operatorname{InSb}(001)-c(8$ $\times 2)$ surface. The rectangle shows the $c(8 \times 2)$ unit mesh. Note the additional intensity on the right-hand side of the $c(8 \times 2)$ unit mesh (marked as $\mathrm{C}$ ), and the rows of bright features (marked as A) aligned along the [110] direction. Diffuse features (marked as D) are also seen between the C features also aligned along the [110] direction. The image was recorded with a sample bias of $+1.1 \mathrm{~V}$ and a tunnel current of $3.2 \mathrm{nA}$.

AIP license or copyright; see http://apl.aip.org/apl/copyright.jsp 

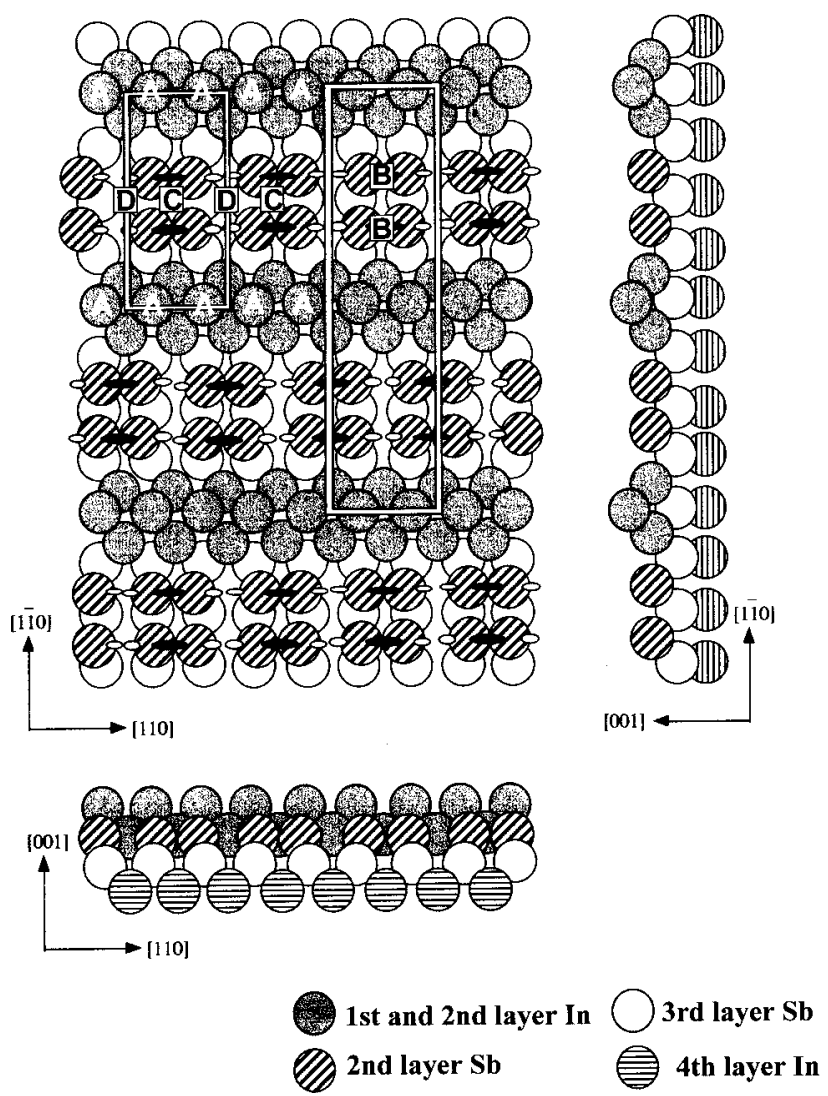

FIG. 3. Plan and side views of the structural model proposed for the Interminated $\operatorname{InSb}(001)-c(8 \times 2)$ surface based on surface $\mathrm{x}$-ray diffraction measurements. Both the $(4 \times 2)$ and $c(8 \times 2)$ unit mesh are also shown and the model is marked with the A, B, C and D features that relate to the filled and empty states images.

the B features indicates that they tend to form pairs by moving slightly toward each other, allowing individual $(4 \times 2)$ blocks to be identified. Within each $(4 \times 2)$ block, four B features are seen, which we have divided into pairs marked by a short line. Schweitzer et al. ${ }^{3}$ had originally attributed these features to electron lone pairs associated with second layer $\mathrm{Sb}$ atoms; however, it is now proposed that these features are due to lone pair orbitals associated with the $\mathrm{Sb}-\mathrm{Sb}$ dimers in the second layer. The two Sb dimer pairs contained within each $(4 \times 2)$ unit in the structural model (marked B) are shown in Fig. 3. The rather even spacing of the B features in Fig. 1 can be attributed to the STM probing the wave functions associated with the $\mathrm{Sb}$ dimers, rather than their nuclear positions.

With the exception of the bright rows of A features along the [110] direction in both STM images, the empty states image in Fig. 2 is markedly different from the filled states image in Fig. 1. The $c(8 \times 2)$ unit mesh has already been identified, but it is somewhat more difficult to identify the individual features in terms of the structural model shown in Fig. 3. We know from the filled state image that there are two $\mathrm{Sb}$ dimer pairs present in each $(4 \times 2)$ block. It is therefore proposed that the "rungs," marked C in Fig. 2, result from empty states associated with these two $\mathrm{Sb}$ dimer pairs. Comparison with empty states images from chemisorbed $\mathrm{Sb}-\mathrm{Sb}$ dimers on the $c(4 \times 4)-\mathrm{InSb}(001)$ surface clearly indicate the presence of these states. ${ }^{4}$ It should also be noted that the empty state image shows the A-C-A features aligned along the [1 $1 \overline{\mathbf{1}} 0]$ direction, whereas the structural model in Fig. 3 shows the $\mathrm{C}$ features lying halfway between two A features in a given row. We attribute this to the fact that the bright rows seen in Fig. 2 are in fact due to a combination of the states associated with In atoms in both the first and second layers. If one considers adjacent A's and the two second layer In atoms between them, and assigns the feature observed in the STM image to a combination of states from that cluster of four metallic bonded In atoms, then repeating this along the In rows indicates that the gaps between the bright $\mathrm{A}$ features seen in the image will lie on a line bisecting the $\mathrm{Sb}-\mathrm{Sb}$ dimers and perpendicular to the dimer direction (as in the model). In addition, the formation of $\mathrm{Sb}-\mathrm{Sb}$ dimers in the second layer (B features) exposes small sections of the third layer in this part of the unit cell (marked D) in both Figs. 2 and 3. This is relevant, since the structural model of Norris and co-workers ${ }^{8}$ has the third layer as a complete $\mathrm{Sb}$ layer. These diffuse features are therefore assigned to empty states associated with the complete $\mathrm{Sb}$ third layer.

In summary, we have presented high resolution filled and empty states STM images of the In-terminated $\operatorname{InSb}(001)-c(8 \times 2)$ surface. These STM images are in excellent agreement with a recently proposed structural model for this surface that represents a significant departure from previously suggested models, e.g., in comparison with the $c(8 \times 2)-\operatorname{GaAs}(001)$ surface reconstruction. Why the $c(8$ $\times 2)-\mathrm{InSb}(001)$ surface should be so different is not known; however, one possibility could be the increased degree of metallicity (i.e., the propensity to form metal-metal bonds) down a given group in the periodic table (hence $\mathrm{In}>\mathrm{Ga}$ ). This could explain why the group III terminated reconstructions for these two surfaces with the same periodicity are so different.

The authors gratefully acknowledge EPSRC for funding the visit to DESY and the E. C. for a research studentship (A.A.D.). Helpful discussions with Professor Colin Norris at Leicester University, UK are also acknowledged.

${ }^{1}$ J. A. Kubby and J. J. Boland, Surf. Sci. Rep. 26, 61 (1996).

${ }^{2}$ Q-K. Xue, T. Hashizume, and T. Sakurai, Prog. Surf. Sci. 56, 1 (1997).

${ }^{3}$ M. O. Schweitzer, F. M. Leibsle, T. S. Jones, C. F. McConville, and N. V. Richardson, Surf. Sci. 280, 63 (1993).

${ }^{4}$ C. F. McConville, T. S. Jones, F. M. Leibsle, S. M. Driver, T. C. Q. Noakes, M. O. Schweitzer, and N. V. Richardson, Phys. Rev. B 50, 14965 (1994).

${ }^{5}$ P. R. Varekamp, M. Bjorkqvist, M. Gothelid, and U. O. Karlsson, Surf. Sci. 350, L221 (1998).

${ }^{6}$ P. John, T. Miller, and T. C. Chang, Phys. Rev. B 39, 1730 (1989).

${ }^{7}$ D. K. Biegelson, R. D. Brigans, J. E. Northrup, and L. E. Schwartz, Phys. Rev. B 41, 5701 (1990).

${ }^{8}$ N. Jones, C. Norris, C. L. Nicklin, P. Steadman, S. H. Baker, A. D. Johnson, and S. L. Bennett, Surf. Sci. 409, 27 (1998). 